University of New Mexico

UNM Digital Repository

Branch Mathematics and Statistics Faculty and Staff Publications

9-30-2003

\title{
Invertibility of current density from near-field electromagnetic data
}

Evangelos A. Coutsias

Daniel Sheltraw

Follow this and additional works at: https://digitalrepository.unm.edu/math_fsp

Part of the Mathematics Commons

\section{Recommended Citation}

Journal of Applied Physics, 94(8): 5307-5315 Article author is part of the Main Campus Math Department.

This Article is brought to you for free and open access by the Branch Academic Departments at UNM Digital Repository. It has been accepted for inclusion in Branch Mathematics and Statistics Faculty and Staff Publications by an authorized administrator of UNM Digital Repository. For more information, please contact disc@unm.edu. 


\title{
Invertibility of current density from near-field electromagnetic data
}

\author{
D. J. Sheltraw ${ }^{\mathrm{a})}$ \\ Department of Radiology, University of New Mexico, 915 Camino de Salud, Albuquerque, \\ New Mexico 87131 \\ E. A. Coutsias \\ Department of Mathematics and Statistics, University of New Mexico, Albuquerque, New Mexico 87131
}

(Received 16 June 2003; accepted 29 July 2003)

\begin{abstract}
The problem of determining a current density confined to a volume from measurements of the magnetic and electric fields it produces exterior to that volume is known to have nonunique solutions. Despite the nonuniqueness of the inversion we show that one may nevertheless uniquely determine certain moments of the vector spherical harmonic expansion of the current. It is demonstrated that the determination of these moments allows for the unique inversion of a current density confined to a spherical shell. Although unique the inversion may be ill conditioned and require a regularization of the inversion as demonstrated in an example numerical inversion.
\end{abstract} (C) 2003 American Institute of Physics. [DOI: 10.1063/1.1611262]

\section{INTRODUCTION}

The electromagnetic inverse problem consists of the determination or estimation of the current source underlying the electric and magnetic fields measured outside the source. However, as was shown by Helmholtz in $1853,{ }^{1}$ a current distribution inside a conductor cannot in general be determined uniquely from knowledge of the electromagnetic field exterior to the conductor. There exist current distributions which give rise to no magnetic field outside, no electric field outside or neither. ${ }^{2,3}$ However, if certain constraints are known to apply to a current distribution, one can perform the inversion uniquely (see-planar case) ${ }^{4}$

In neuroscience applications one is usually interested in obtaining information about the primary current source $\mathbf{J}^{p}$ due to direct neuronal activity and defined in the following decomposition of the total current: $\mathbf{J}=\mathbf{J}^{p}+\sigma \mathbf{E}$. One is then faced with the problem of inverting for both $\mathbf{J}^{p}$ and $\mathbf{E}$ simultaneously given external field data. This task is further complicated by the difficulty of performing detailed measurements of tissue conductivity $\sigma(\mathbf{x}) \cdot{ }^{5-7}$ In the case of an infinite homogeneous conductivity the ohmic currents, $\sigma(\mathbf{x}) \mathbf{E}$, make no contributions to the external magnetic field, which can then be expressed in terms of the primary current alone. Of course, the essential non-uniqueness of the inversion remains ${ }^{8}$ requiring additional constraints or assumptions for a unique inversion. 8,9

The purpose of this article is twofold: (1) to give a complete characterization of those quantities related to the current density which can be determined by a linear inversion and (2) to present a numerical algorithm for the unique inversion on a spherical shell.

We state the idealized near-field electromagnetic inverse problem as follows: Given the magnetic field $\mathbf{B}(\mathbf{r}, t)$ and the electric field $\mathbf{E}(\mathbf{r}, t)$, known everywhere on a spherical shell

a) Author to whom correspondence should be addressed; electronic mail: sheltraw@unm.edu and due to the current source $\mathbf{J}(\mathbf{r}, t)$ contained in the interior of the shell, invert the field data to obtain the current density or some of its properties. Initially we do not invoke the infinite homogeneous conductor assumption and we seek instead inversion for the more general problem of total (primary plus ohmic) current. We take this approach since there is considerable dispute over the applicability of the infinite homogeneous conductor and spherically symmetric conductor assumptions to most situations of practical interest. ${ }^{5}$ However we do contrast our results to the infinite homogeneous conductor model since it is a useful idealization. ${ }^{5-7}$

The article is organized as follows: In Sec. II we give a consistent presentation of the near-field approximation as pertains to an inversion for the total current density. Section III investigates the uniquely determined properties of a current density for the inverse magnetometry problem. Section IV shows in what manner electrical potential data may add to the inversion problem. Section $\mathrm{V}$ then makes connection with the infinite homogeneous conductor case. Section VI applies the results of the preceding sections to the unique inversion of a current on a spherical shell. Although unique, the inversion is still not well-posed in the Hadamard sense since, as will be shown, the inversion can be ill-conditioned leading to "high frequency" noise amplification. Regularization may be needed to make the inversion well-behaved. In Sec. VII we formulate a simulated experiment to illustrate the competing effects of increased magnetic field sampling, noise amplification, and regularization. Conclusions appear in Sec. VIII.

\section{A CONSISTENT NEAR-FIELD APPROXIMATION}

Although Plonsey ${ }^{3}$ has treated with success the quasistatic problem for primary currents by neglecting certain time derivatives in the Maxwell equations, such an approximation leads to inconsistency if applied to the total current situation. Namely the neglect of the displacement current $(1 / c)(\partial \overline{\mathbf{e}} / \partial t)$ implies that $\boldsymbol{\nabla} \cdot \overline{\mathbf{j}}=0$. But since the charge distri- 
bution $\bar{q}$ is initially zero the equation $\boldsymbol{\nabla} \cdot \overline{\mathbf{j}}=0$ implies, through the equation of continuity, that it will remain zero as will the electric potential. Clearly an approach focused on the total current must account for the near-field electric potentials which are measured routinely in the low-frequency electroencephalography experiment.

In this section we derive a consistent near field approximation to form the basis of the inverse problem for the total current. The microscopic Maxwell equations are written as

$$
\begin{aligned}
& \boldsymbol{\nabla} \cdot \mathbf{b}(\mathbf{x}, t)=0 \quad \boldsymbol{\nabla} \cdot \mathbf{e}(\mathbf{x}, t)=4 \pi q(\mathbf{x}, t), \\
& \boldsymbol{\nabla} \times \mathbf{b}(\mathbf{x}, t)-\frac{1}{c} \frac{\partial}{\partial t} \mathbf{e}(\mathbf{x}, t)=\frac{4 \pi}{c} \mathbf{j}(\mathbf{x}, t), \\
& \boldsymbol{\nabla} \times \mathbf{e}(\mathbf{x}, t)+\frac{1}{c} \frac{\partial}{\partial t} \mathbf{b}(\mathbf{x}, t)=0 .
\end{aligned}
$$

It will be assumed that there exists no initial charge distribution despite the fact that charge separation does exist across a resting polarized neuron. To do so assumes a spatial scale of interest which we take to be a volume $V$ inclusive of many neurons (possibly a cubic millimeter) over which the average charge is zero. Such an average denoted by $\bar{f}(\mathbf{x}, t)$ for a microscopic quantity $f(\mathbf{x}, t)$ is defined by $\bar{f}(\mathbf{x}, t)=1 / V \int_{V} f(\mathbf{x}$ $\left.+\mathbf{x}^{\prime}, t\right) d^{3} \mathbf{x}^{\prime}$. Since this averaging process is linear we may then write the macroscopic equations at this scale of interest to simply be

$$
\begin{aligned}
& \boldsymbol{\nabla} \cdot \overline{\mathbf{b}}(\mathbf{x}, \omega)=0 \quad \nabla \cdot \overline{\mathbf{e}}(\mathbf{x}, \omega)=4 \pi \bar{q}(\mathbf{x}, \omega), \\
& \nabla \times \overline{\mathbf{b}}(\mathbf{x}, \omega)+i \omega / c \overline{\mathbf{e}}(\mathbf{x}, \omega)=\frac{4 \pi}{c} \overline{\mathbf{j}}(\mathbf{x}, \omega), \\
& \nabla \times \overline{\mathbf{e}}(\mathbf{x}, \omega)-\frac{i \omega}{c} \overline{\mathbf{b}}(\mathbf{x}, \omega)=0
\end{aligned}
$$

where we have also transformed into frequency space to benefit from the change of time derivatives to algebraic quantities. In the remainder of this paper we will drop the explicit frequency dependence of vector and scalar fields.

In terms of the vector potential $\overline{\mathbf{a}}(\mathbf{x})$ and scalar potential $\bar{\phi}(\mathbf{x})$ the Maxwell equations may be written in the Lorentz gauge as

$$
\begin{aligned}
& \nabla^{2} \overline{\mathbf{a}}(\mathbf{x})+\frac{\omega^{2}}{c^{2}} \overline{\mathbf{a}}(\mathbf{x})=-\frac{4 \pi}{c} \overline{\mathbf{j}}(\mathbf{x}), \\
& \nabla^{2} \bar{\phi}(\mathbf{x})+\frac{\omega^{2}}{c^{2}} \bar{\phi}(\mathbf{x})=\frac{i 4 \pi}{\omega} \nabla \cdot \overline{\mathbf{j}}(\mathbf{x}),
\end{aligned}
$$

where the potentials are defined by

$$
\begin{aligned}
& \overline{\mathbf{b}}(\mathbf{x})=\nabla \times \overline{\mathbf{a}}(\mathbf{x}), \\
& \frac{i \omega}{c} \overline{\mathbf{a}}(\mathbf{x})-\overline{\mathbf{e}}(\mathbf{x})=\nabla \bar{\phi}(\mathbf{x})
\end{aligned}
$$

and the Lorentz gauge is given by

$$
\boldsymbol{\nabla} \cdot \overline{\mathbf{a}}(\mathbf{x})=\frac{i \omega}{c} \bar{\phi}(\mathbf{x})
$$

The frequencies involved in neuronal activity (typically $\approx 100 \mathrm{~Hz})^{8}$ are small compared to the reciprocal of the time needed for the signal to propagate from the source to the sensor. This leads to a near-field expansion with respect to the small dimensionless parameter $\epsilon=R \Omega_{c} / c$, the ratio of a characteristic distance $R$ between the source positions and the positions of the field measurements (approximately 25 $\mathrm{cm}$ ) to the distance that would be propagated by the electromagnetic field in vacuum during a characteristic period. Here $\Omega_{c}$ is the characteristic frequency of the current density. To accomplish this Eqs. (3)-(4) are written in terms of the dimensionless independent variables $\mathbf{r}=\mathbf{x} / R, w=\omega / \Omega_{c}$, and the dimensionless dependent variables $\mathbf{J}=\overline{\mathbf{j}} / J_{c}, \quad \mathbf{A}$ $=c \overline{\mathbf{a}} / J_{c} 4 \pi R^{2}, \quad \Phi=c \bar{\phi} / J_{c} 4 \pi R^{2}, \quad \mathbf{E}=c \overline{\mathbf{e}} / J_{c} 4 \pi R, \quad$ and $\quad \mathbf{B}$ $=c \overline{\mathbf{b}} / J_{c} 4 \pi R$ as

$$
\begin{aligned}
& \nabla^{2} \mathbf{A}(\mathbf{r})+\epsilon^{2} w^{2} \mathbf{A}(\mathbf{r})=-\mathbf{J}(\mathbf{r}) \\
& \epsilon \nabla^{2} \Phi(\mathbf{r})+\epsilon^{3} w^{2} \Phi(\mathbf{r})=\frac{i}{w} \nabla \cdot \mathbf{J}(\mathbf{r}),
\end{aligned}
$$

where $J_{c}$ is a characteristic current magnitude. The definitions of the potentials Eq. (5) and Lorentz gauge Eq. (6) become

$$
\begin{aligned}
& \mathbf{B}(\mathbf{r})=\boldsymbol{\nabla} \times \mathbf{A}(\mathbf{r}) \quad i \epsilon w \mathbf{A}(\mathbf{r})-\mathbf{E}(\mathbf{r})=\nabla \Phi(\mathbf{r}), \\
& \boldsymbol{\nabla} \cdot \mathbf{A}(\mathbf{r})=i \epsilon w \Phi(\mathbf{r}) .
\end{aligned}
$$

We obtain a near-field approximation by expanding the magnetic, electric, and current fields in terms of the smallness parameter $\epsilon$ as follows:

$$
\begin{aligned}
& \mathbf{A}(\mathbf{r} ; \epsilon)=\sum_{n} \epsilon^{n} \mathbf{A}_{n}(\mathbf{r}) \quad \Phi(\mathbf{r} ; \epsilon)=\sum_{n} \epsilon^{n} \Phi_{n}(\mathbf{r}), \\
& \mathbf{J}(\mathbf{r} ; \epsilon)=\sum_{n} \epsilon^{n} \mathbf{J}_{n}(\mathbf{r}) .
\end{aligned}
$$

Substitution of Eq. (12) into Eqs. (7)-(10) yields, upon collecting the zeroth order contributions, the following set of equations:

$$
\begin{aligned}
& \nabla^{2} \mathbf{A}_{0}=-\mathbf{J}_{0} \quad \mathbf{E}_{0}=-\nabla \Phi_{0} \\
& \boldsymbol{\nabla} \cdot \mathbf{J}_{0}=0 \quad \mathbf{B}_{0}=\boldsymbol{\nabla} \times \mathbf{A}_{0} \\
& \boldsymbol{\nabla} \cdot \mathbf{A}_{0}=0
\end{aligned}
$$

Similarly, the first order contributions yield the additional set of equations

$$
\begin{aligned}
& \nabla^{2} \mathbf{A}_{1}=-\mathbf{J}_{1} \quad \nabla^{2} \Phi_{0}=\frac{i}{w} \boldsymbol{\nabla} \cdot \mathbf{J}_{1}, \\
& \mathbf{B}_{1}=\boldsymbol{\nabla} \times \mathbf{A}_{1} \quad i w \mathbf{A}_{0}-\mathbf{E}_{1}=\nabla \Phi_{1}, \\
& \nabla \cdot \mathbf{A}_{1}=i w \Phi_{0} .
\end{aligned}
$$

The solutions of the potential equations given in Eqs. (13) and (16) are, respectively,

$\mathbf{A}_{n}(\mathbf{r})=\frac{1}{4 \pi} \int \frac{\mathbf{J}_{n}\left(\mathbf{r}^{\prime}\right)}{\left|\mathbf{r}-\mathbf{r}^{\prime}\right|} d^{3} \mathbf{r}^{\prime}$, 


$$
\Phi_{0}(\mathbf{r})=-\frac{i}{4 \pi w} \int \frac{\nabla^{\prime} \cdot \mathbf{J}_{1}\left(\mathbf{r}^{\prime}\right)}{\left|\mathbf{r}-\mathbf{r}^{\prime}\right|} d^{3} \mathbf{r}^{\prime},
$$

where $n=0,1$. The expansion of the current density in the smallness parameter $\epsilon$ shows the dependence of the current on the characteristic distance $R$. That dependence is implicit in the confinement of the current within a volume of radius $R$.

\section{MAGNETIC FIELD DATA AND INVERSION}

In this section we investigate the determinable quantities of the inverse magnetometry problem. Since the measurements are performed on a spherical shell it is natural to expand the magnetic field (and vector potential) in terms of vector spherical harmonics (VSH). The resulting expressions will allow a precise characterization of the properties of the current density which the idealized magnetometry experiment may determine. External to the current containing region we may write Eq. (19) with $n=0$ as ${ }^{10}$

$$
\mathbf{A}_{0}(\mathbf{r})=\sum_{l j m}\left(\int_{V} \frac{r^{\prime l}}{2 l+1} \mathbf{J}_{0}\left(\mathbf{r}^{\prime}\right) \cdot \mathbf{Y}_{j m}^{* l}\left(\Omega^{\prime}\right) d^{3} \mathbf{r}^{\prime}\right) \frac{\mathbf{Y}_{j m}^{l}(\Omega)}{r^{(l+1)}}
$$

where $l=j, j+1, j-1$ (with the exception that $l=1$ for $j$ $=0)$, and $m=-j,-j+1, \ldots, j-1, j$. We will, as in the above equation, use the variable $\Omega$ to denote the ordered pair of angular variables $(\theta, \phi)$. By writing the zeroth order current in the VSH expansion $\mathbf{J}_{0}(\mathbf{r})=\Sigma_{l j m} a_{j m}^{l}(r) \mathbf{Y}_{j m}^{l}(\Omega)$ Eq. (21) becomes

$$
\mathbf{A}_{0}(\mathbf{r})=\sum_{l j m}\left(\frac{m_{j m}^{l l}}{2 l+1}\right) \frac{\mathbf{Y}_{j m}^{l}(\Omega)}{r^{l+1}}
$$

where we have defined $\mathrm{m}_{j m}^{l k}=\int_{0}^{1} r^{k} a_{j m}^{l}(r) r^{2} d r$. Now since $\boldsymbol{\nabla} \cdot \mathbf{J}_{0}(\mathbf{r})=0$ it can easily be shown (see Appendix A) that $m_{j m}^{j-1, j-1}=0$. Using this property and taking the curl of Eq. (22) gives the magnetic field on the unit shell surface

$$
\mathbf{B}_{0}(\Omega)=-i \sum_{j m}\left(\frac{j}{2 j+1}\right)^{1 / 2} m_{j m}^{j j} \mathbf{Y}_{j m}^{j+1}(\Omega) .
$$

Since we are interested in determining the moments $m_{j m}^{j j}$ we can simply write Eq. (23) in terms of the radial component of $\mathbf{B}_{0} \cdot \mathbf{e}_{r}=B_{0 r}$ only to obtain

$$
B_{0 r}(\Omega)=i \sum_{j m} \frac{j^{1 / 2}(j+1)^{1 / 2}}{2 j+1} m_{j m}^{j j} Y_{j m}(\Omega)
$$

and, transforming back to the time domain, we arrive at the result of this section

$$
\begin{aligned}
& m_{j m}^{j j}(t)=-i \frac{2 j+1}{j^{1 / 2}(j+1)^{1 / 2}} B_{0 r}^{j m}(t), \\
& m_{j m}^{j-1, j-1}(t)=0,
\end{aligned}
$$

where we have defined $B_{0 r}^{j m}=\int B_{0 r}(\Omega) Y_{j m}^{*}(\Omega) d \Omega$. In Eq. (25) the $j=0$ term poses no special problem since $\mathbf{Y}_{00}^{0}(\Omega)$ $=0$.

From Eq. (23) we can conclude that the $l=j+1, j-1$ components of the current are always silent in this lowest order near-field approximation since they do not contribute to the magnetic field. Furthermore, any current with vanishing moments $m_{j m}^{j j}$ is as well a silent current. From Eq. (25) we may conclude that the measurement of the magnetic field can only give information about the $l=j$ component of the current density while the $\boldsymbol{\nabla} \cdot \mathbf{J}_{0}=0$ constraint yields a restriction upon the $l=j-1$ component of the current density. No information about the $l=j+1$ component of the current density is obtained from the field measurement without additional constraints being placed upon the current.

\section{ELECTRIC FIELD DATA AND INVERSION}

Electric potential measurements give information about the small but, as we will see, not necessarily negligible $\mathbf{J}_{1}$. The zeroth order solution for the electric potential given by Eq. (20) can be written as ${ }^{10}$

$$
\begin{aligned}
\Phi_{0}(\mathbf{r})= & -\frac{i}{w} \sum_{j m} \int \frac{\nabla^{\prime} \cdot \mathbf{J}_{1}\left(\mathbf{r}^{\prime}\right)}{2 j+1} \\
& \times \frac{r^{\prime j}}{r^{j+1}} Y_{j m}^{*}\left(\Omega^{\prime}\right) Y_{j m}(\Omega) d^{3} \mathbf{r}^{\prime} .
\end{aligned}
$$

Substituting the VSH expansion, $\mathbf{J}_{1}=\Sigma_{l j m} \alpha_{j m}^{l}(r) \mathbf{Y}_{j m}^{l}(\Omega)$, into Eq. (26) and simplifying we obtain

$$
\Phi_{0}(\mathbf{r})=\frac{i}{w} \sum_{j m} \mu_{j m}^{j-1, j-1} Y_{j m}(\Omega),
$$

where we have used $\mathbf{n} \cdot \mathbf{J}_{1}=0$ on the surface of the unit sphere and we have defined $\mu_{j m}^{l k}=\int_{0}^{1} r^{k} \alpha_{j m}^{l}(r) r^{2} d r$. Defining $\Phi_{j m}=\int \Phi_{0}(\mathbf{r}) Y_{j m}^{*}(\Omega) d \Omega$, where the integral is taken over the unit sphere, Eq. (27) can be written as

$$
\mu_{j m}^{j-1, j-1}=-i w \Phi_{j m}\left(\frac{2 j+1}{j}\right)^{1 / 2} .
$$

Also, since $\mathbf{J}_{1}$ obeys Eq. (20) one can write, as for $\mathbf{J}_{0}$, that

$$
\mu_{j m}^{j j}=-i \frac{2 j+1}{j^{1 / 2}(j+1)^{1 / 2}} B_{1 r}^{j m} .
$$

Transforming into the time domain we can now write the result of this section as

$$
\begin{aligned}
& \mu_{j m}^{j j}(t)=-\mathrm{i} \frac{2 j+1}{j^{1 / 2}(j+1)^{1 / 2}} B_{1 r}^{j m}(t), \\
& \mu_{j m}^{j-1, j-1}(t)=\left(\frac{2 j+1}{j}\right)^{1 / 2} \frac{\partial \Phi_{j m}}{\partial t} .
\end{aligned}
$$

Although electric potential measurements only give information about the relatively small $\epsilon \mathbf{J}_{1}$ contribution to the current, this information is not necessarily negligible. Such is the case for the $l=j-1$ component of the current density for which the zeroth order magnetic field measurements give no direct information. Although knowledge of the small first order current [obtained from Eq. (31)] contributes little to the total current magnitude, it may provide localization information. In addition it should be noted that the $\mathbf{J}_{1}$ contribution to the current is notably different from the $\mathbf{J}_{0}$ contribution in that the former leads to a net charge formation (the diver- 
gence is non zero) and therefore may give information of a special nature albeit of smaller amplitude than the $\mathbf{J}_{0}$ term. Note that Eq. (31) provides no significant additional information about the $l=j$ component of the current density since $B_{1 r}$ is negligible compared $B_{0 r}$ and cannot be measured independent of $B_{0 r}$.

\section{INFINITE HOMOGENEOUS CONDUCTOR}

In this section we make connections with the infinite homogeneous conductor model. If one has knowledge of the conductivity of the medium containing the primary current sources one may write $\mathbf{J}=\mathbf{J}_{p}-\sigma \nabla \Phi$, where $\mathbf{J}_{p}$ is the primary current source and $-\sigma \nabla \Phi$ is the current given by Ohm's law. Note that this expression uses the dimensionless conductivity constant $\sigma=4 \pi \bar{\sigma} R J_{c} / c$, where $\sigma$ is the conductivity averaged over a suitable spatial scale. This relationship is valid for all orders of the expansion in the smallness parameter $\epsilon$ so that

$$
\mathbf{J}_{0}(\mathbf{r})=\mathbf{J}_{0 p}(\mathbf{r})-\sigma \nabla \Phi_{0}(\mathbf{r}) .
$$

Strictly speaking we should include the contribution of effective current densities from magnetization and polarization effects. However, these currents make a negligible contribution to the total current as shown by Plonsey. ${ }^{3}$ If the conductivity $\sigma$ can be considered to be infinite homogeneous this form of the total current density, when combined with Eqs. (13) and (16), yields the following connection between zeroth and first order contributions to the current density:

$$
\nabla \cdot \mathbf{J}_{1}(\mathbf{r})=\frac{i w}{\sigma} \nabla \cdot \mathbf{J}_{0 p}(\mathbf{r})
$$

Substitution of Eqs. (32) and (33) into Eq. (20) then gives

$$
\begin{aligned}
& \mathbf{A}_{0}(\mathbf{r})=\frac{1}{4 \pi} \int \frac{\mathbf{J}_{0 p}\left(\mathbf{r}^{\prime}\right)}{\left|\mathbf{r}-\mathbf{r}^{\prime}\right|}, \\
& \Phi_{0}(\mathbf{r})=-\frac{1}{4 \pi \sigma} \int \frac{\nabla^{\prime} \cdot \mathbf{J}_{0 p}\left(\mathbf{r}^{\prime}\right)}{\left|\mathbf{r}-\mathbf{r}^{\prime}\right|} d^{3} \mathbf{r}^{\prime} .
\end{aligned}
$$

As noted by others ${ }^{3}$ the ohmic contribution to the current density does not contribute to the magnetic field in this case of a homogeneous conducting medium. Note that Eqs. (34) and (35) both depend on the zeroth order primary current only.

A derivation similar to those presented in the preceding sections of this article results in the uniquely determined quantities

$$
\begin{aligned}
& m_{j m}^{j j}(t)=-\mathrm{i} \frac{2 j+1}{j^{1 / 2}(j+1)^{1 / 2}} B_{0 r}^{j m}(t), \\
& m_{j m}^{j-1, j-1}(t)=\sigma\left(\frac{2 j+1}{j}\right)^{1 / 2} \Phi_{j m}(t),
\end{aligned}
$$

where $m_{j m}^{l k}(t)=\int_{0}^{1} r^{k} a_{j m}^{l}(r, t) r^{2} d r$ and the $a_{j m}^{l}(r, w)$ are the VSH coefficients of the zeroth order primary current $\mathbf{J}_{0 p}$. Therefore, in the case of the homogeneous conductor the electric and magnetic field measurements yield zeroth order current density information in orthogonal subspaces. Notice that, as in the total current case, there is no information ob- tained about the $l=j+1$ components of the current density. Also notice that, unlike the total current case, the determinable quantities $m_{j m}^{j-1, j-1}$ depend on the electric potential rather than its time derivative.

\section{A UNIQUELY INVERTIBLE CURRENT DENSITY}

We now look at a case where the magnetic and electric field information is enough to determine the current density. In the following we assume that the current is restricted to a shell of radius $r_{0}$. Results are given for both the total current and the primary current of an infinite homogeneous conductor. Although the infinite homogeneous conductor is rarely realized as a suitable approximation to the practical problems of interest this does serve as an important case with which to draw distinction with the total current case.

\section{A. Total current case}

In this case $\mathbf{J}=\mathbf{J}_{0}+\epsilon \mathbf{J}_{1}$ and we may write the coefficients of the VSH expansion of the current density as

$$
\mathrm{a}_{j m}^{l}(r)=r^{-2} \delta\left(r-r_{0}\right) b_{j m}^{l}, \quad \alpha_{j m}^{l}=r^{-2} \delta\left(r-r_{0}\right) \beta_{j m}^{l},
$$

where $l=j, j+1, j-1$ and the coefficients $b_{j m}^{j}$ and $\beta_{j m}^{j}$ are to be determined from measurement of the moments $m_{j m}^{j}$ and $\mu_{j m}^{j-1, j-1}$. Multiplying each side of Eq. (38) by the appropriate power of $r$ and integrating we obtain

$$
m_{j m}^{l k}(r)=r_{0}^{k} b_{j m}^{l}, \quad \mu_{j m}^{l k}=r_{0}^{k} \beta_{j m}^{l} .
$$

One can view the solution of Eq. (39) as a simple inversion of a diagonal matrix. However, the dependence on $r_{0}$ may make these diagonal matrices ill conditoned with condition number given by $r_{0}^{1-j_{c}}$, where $j_{c}$ is a cutoff number for $j$. As a result noise in the high spatial frequency components of the current density may be amplified. To obtain a well-conditioned inversion we apply a Tikhonov regularization (see Appendix D) to smooth the high frequency noise. For a diagonal matrix the classical Tikhonov regularization takes the simple form

$$
b_{j m}^{l}=\frac{r_{0}^{k}}{r_{0}^{2 k}+\lambda^{2}} m_{j m}^{l k}(r), \quad \beta_{j m}^{l}=\frac{r_{0}^{k}}{r_{0}^{2 k}+\lambda^{2}} \mu_{j m}^{l k},
$$

where $\lambda$ is the regularization parameter and we have set $f^{\infty}$ $=0$ and $L=I$ (see Appendix D). A proper choice of the regularization parameter $\lambda$ balances spatial smoothing against noise reduction.

The restriction of the current to the shell surface combined with $\boldsymbol{\nabla} \cdot \mathbf{J}_{0}=0$ implies that $\mathbf{J}_{0} \cdot \mathbf{r}=0$. This condition gives the following relationship between the $l=j+1$ and $l$ $=j-1$ components:

$$
a_{j m}^{j+1}=\sqrt{\frac{j}{j+1}} a_{j m}^{j-1}, \quad \alpha_{j m}^{j+1}=\sqrt{\frac{j}{j+1}} \alpha_{j m}^{j-1} .
$$

Combining the definition of the known quantities $m_{j m}^{j j}$, $m_{j m}^{j-1, j-1}$, and $\mu_{j m}^{j-1, j-1}$ with Eq. (41) one then obtains the current density 


$$
\begin{aligned}
\mathbf{J}= & \delta\left(r-r_{0}\right) \sum_{j m}^{j_{c}}\left[m_{j m}^{j j} \frac{r_{0}^{j-2}}{r_{0}^{2 j}+\lambda^{2}} \mathbf{Y}_{j m}^{J}\right. \\
& \left.+\epsilon \sqrt{j} \mu_{j m}^{j-1, j-1} \frac{r_{0}^{j-3}}{r_{0}^{2 j-2}+\lambda^{2}}\left(\frac{\mathbf{Y}_{j m}^{j-1}}{\sqrt{j}}+\frac{\mathbf{Y}_{j m}^{j+1}}{\sqrt{j+1}}\right)\right]
\end{aligned}
$$

or in terms of the magnetic and electric field quantities

$$
\begin{aligned}
\mathbf{J}= & \delta\left(r-r_{0}\right) \sum_{j m}^{j_{c}}\left[-i B_{0 r}^{j m} \frac{2 j+1}{\sqrt{j^{2}+j}} \frac{r_{0}^{j-2}}{r_{0}^{2 j}+\lambda^{2}} \mathbf{Y}_{j m}^{j}\right. \\
& \left.+\epsilon \sqrt{2 j+1} \frac{\partial \Phi_{j m}}{\partial t} \frac{r_{0}^{j-3}}{r_{0}^{2 j-2}+\lambda^{2}}\left(\frac{\mathbf{Y}_{j m}^{j+1}}{\sqrt{j}}+\frac{\mathbf{Y}_{j m}^{j-1}}{\sqrt{j+1}}\right)\right] .
\end{aligned}
$$

\section{B. Infinite homogeneous conductor case}

Now we consider the case of an infinite homogeneous conductor. In this case we write the coefficients of the VSH expansion of the current density as

$$
a_{j m}^{l}(r)=r^{-2} \delta\left(r-r_{0}\right) b_{j m}^{l},
$$

where again the $b_{j m}^{l}$ are coefficients to be determined. If we assume that $\mathbf{J}_{p} \cdot \mathbf{r}=0$ we may write, in a manner similar to the total current,

$$
\begin{aligned}
\mathbf{J}_{0 p}= & \delta\left(r-r_{0}\right) \sum_{j m}\left[-i B_{0 r}^{j m} \frac{2 j+1}{\sqrt{j^{2}+j}} \frac{r_{0}^{j-2}}{r_{0}^{2 j}+\lambda^{2}} \mathbf{Y}_{j m}^{j}\right. \\
& \left.+\sigma \sqrt{2 j+1} \Phi_{j m} \frac{r_{0}^{j-3}}{r_{0}^{2 j-2}+\lambda^{2}}\left(\frac{\mathbf{Y}_{j m}^{j+1}}{\sqrt{j}}+\frac{\mathbf{Y}_{j m}^{j-1}}{\sqrt{j+1}}\right)\right] .
\end{aligned}
$$

Note that the condition $\mathbf{J}_{p} \cdot \mathbf{r}=0$ is an additional constraint which is not implied by restriction to the shell as in the total current case.

\section{Validity of inversion}

Having found the VSH expansion of the current densities of Secs. VI A and VIB from measurements of the magnetic and electric fields does not guarantee that the series given by Eq. (43) or (45) will converge. If we insist that the currents have finite energy, then it is appropriate to require that $\|\mathbf{J}\|_{2}<\infty$; for the case in Eq. (43) without electric field contributions, e.g., this implies

$$
\|\mathbf{J}\|_{2}^{2}=\sum_{j m} \frac{\left[B_{0 r}^{j m}(2 j+1)\right]^{2}}{r_{0}^{2 j+4} j(j+1)}<\infty .
$$

Clearly, this restricts the magnetic field, and the smaller we desire $r_{0}$ to be for the shell on which we perform the inversion, the more severe the restriction. The above condition is of course met whenever the radius of reconstruction, $r_{0}$, is greater than or equal to the radius of a sphere completely enclosing the current, $r_{c}$. But reconstruction on a spherical shell of smaller radius $r_{0}<r_{c}$ could be also found for sufficiently fast decaying $B_{0 r}^{j m}$. On the other hand, for regularized data such as that associated with a truncated spherical harmonic expansion, a reconstruction is guaranteed for arbitrary $r_{0}>0$.

\section{A SIMULATED INVERSION ON A SPHERICAL SHELL}

In this section a simulation to test the inversion on a spherical conducting shell is presented. In the next section we will use this simulation in a number of examples to test the inversion method on the shell. We assume the current density to be restricted to a shell of known radius $r_{0}<1$. We also assume that the radial component of the magnetic field, $B_{r}$, can be sampled on a grid (to be specified below) of a larger spherical shell of radius $r=1$. We will make use of the SPHEREPACK-3.0 Fortran-77 code $^{11,12}$ to perform the analysis of a simulated magnetic field to find the values $B_{0 r}^{j m}$ and the subsequent synthesis of the current density.

\section{A. The simulation}

The $\mathbf{J}_{0}$ part of the current density given by Eq. (43) may be reconstructed according to

$$
\mathbf{J}=-i \delta\left(r-r_{0}\right) \sum_{j m}^{j_{c}} B_{0 r}^{j m} \frac{2 j+1}{\sqrt{j^{2}+j}} \frac{r_{0}^{j-2}}{r_{0}^{2 j}+\lambda^{2}} \mathbf{Y}_{j m}^{j} .
$$

In the case of the current shell it is somewhat simpler to represent the results of the inversion in terms of a scalar stream function $\psi(\theta, \phi)$ such that $\mathbf{J}(\mathbf{r})=r^{-2} \delta\left(r-r_{0}\right) \mathbf{e}_{r}$ $\times \nabla_{\Omega} \psi$ where

$$
\psi=\sum_{j m} B_{0 r}^{j m} \frac{r_{0}^{j}}{r_{0}^{2 j}+\lambda^{2}} \frac{2 j+1}{j(j+1)} Y_{j m} .
$$

To create simulated magnetic field measurements we make use of a current density of the form

$$
\begin{aligned}
& \mathbf{J}_{0}=\mathbf{e}_{\phi} \frac{\delta\left(r-r_{0}\right)}{r_{0}^{2}} \sin \theta G(\theta), \\
& G(\theta)=\sum_{n=0}^{N_{0}} \frac{2 n+1}{2} P_{n}\left(\cos \theta_{0}\right) P_{n}(\cos \theta),
\end{aligned}
$$

where the $P_{n}$ are Legendre functions and the function $G(\theta)$ is a truncated Legendre expansion of $\delta\left(\cos \theta-\cos \theta_{0}\right)$.

The radial component of the magnetic field due to the currents of the type given in Eq. (49) is directly calculated as

$$
\begin{aligned}
B_{r} & =\sum_{l=1}^{N_{0}} \alpha_{l} P_{l}(\cos \theta), \\
\alpha_{l} & =\frac{r_{0}^{l+1}}{4 \pi} \frac{l(l+1)}{2 l+1}\left[P_{l+1}\left(\cos \theta_{0}\right)-P_{l-1}\left(\cos \theta_{0}\right)\right],
\end{aligned}
$$

where we have taken $r=1$. The field due to these elemental currents can be rotated by the angles $\left(\theta_{r}, \phi_{r}\right)$ (see Appendix C) and superimposed upon others to give a richer set of simulated fields to test the inversion algorithm and its limitations. In the next section of this article we will use these simulated fields to test the inversion method on the shell.

We also add noise to the simulated field to test the stability of the inversion and our ability to eliminate instability 
through regularization. We do so by adding noise of a given signal-to-noise ratio (SNR) to each field point in the simulated measurement. The $\mathrm{SNR}$ is given by $\mathrm{SNR}=S_{\max } \sigma$, where $S_{\max }$ is the maximum signal over all field points and $\sigma$ is the standard deviation of 0 deviates. ${ }^{13}$

For the purpose of error analysis we combine Eqs. (48), (50), and (51) to obtain the current stream function directly as

$$
\psi=\frac{r_{0}}{4 \pi} \sum_{n=1}^{N_{0}} P_{n}(\cos \theta)\left[P_{n+1}\left(\cos \theta_{0}\right)-P_{n-1}\left(\cos \theta_{0}\right)\right],
$$

which will be useful in the next section of this article.

As discussed by Swarztrauber, ${ }^{11,12}$ the analysis of $B_{0 r}$ into spherical harmonic components $B_{0 r}^{j m}$ requires truncations with $j \leqslant N$ the number of zonal harmonics included. For each $j$ there are $2 j+1$ values for $m$, therefore, the determination of $(N+1)^{2}$ spherical harmonic coefficients is required. On the other hand, the spherical transform algorithms given in SPHEREPACK-3.0 require sampling on a grid that is equally spaced in both longitude $\phi$ and latitude $\theta$. With the product grid containing $N$ points in the $\theta$ direction and $2 N$ points in the $\phi$ direction, this results in $2 N^{2}$ data points, making the analysis problem 1 . This results in the analysis being performed as a least squares problem. ${ }^{11,14}$ A spectral filtering where at $\theta=\theta_{i}$ only $2 N \sin \theta_{i}$ longitudinal points are required can be employed without effective loss of resolution. ${ }^{14}$ This reduces the number of required sample values to $\pi N^{2} / 2$ for the entire sphere although the analysis problem is still 1. To employ the above algorithm, our method requires the values on the reduced grid and produces the values on the equispaced grid (used by SPHEREPACK-3.0) by fast Fourier transform based interpolation.

\section{B. An example}

In this section we give an example inversion to test the ability to resolve currents on a spherical shell and explore the amount of regularization needed to control the instability discussed in Sec. VI. The example demonstrates the effect of variation in the following parameters: The number of latitude and longitude sampling points, $n_{\text {lat }}$ and $n_{\text {lon }}$; the SNR, and the regularization parameter $\lambda$.

In Figs. 1-6 we show the graphical results of an example inversion for two closely spaced current rings on a spherical shell of radius $r_{0}=0.8$ which is the reconstruction surface as well. Figure 1 is a direct plot of the current stream function as given by Eq. (52). The plots of Figs. 1-2 can be compared to this direct plot for a visual check of inversion accuracy. Tables I and II give a more precise check of the inversion error.

Figures 2 and 3 pertain to a noiseless sampling of the magnetic field. These figures illustrate the progressively better resolution of the current density as sampling of the magnetic field is increased. Table I shows a precipitous reduction in the error near a sampling such that $n_{\text {lat }}=41$ and $n_{\text {lon }}$ $=80$. Although increasing the sampling of the field allows one to compute components of the current stream function of larger $j$ and $m$ values and therefore provide greater detail in the inversion this trend does not persist as one can see when

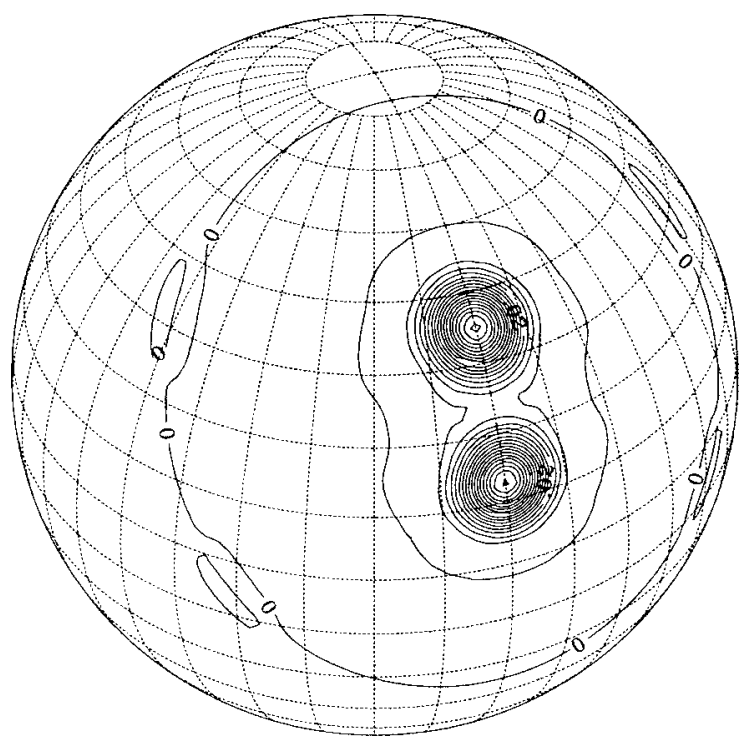

FIG. 1. Direct plot of the current stream function with $N_{n 1}=35,\left(\theta_{r}, \phi_{r}\right)_{1}$ $=(45,0), N_{n 2}=35,\left(\theta_{r}, \phi_{r}\right)_{2}=(67,0)$.

the sampling is near $n_{\text {lat }}=49$ and $n_{\text {lon }}=96$. Near this sampling density an amplification of machine precision "noise," due to the ill-conditioned nature of the inversion, begins to compete with the reduction in error due to increased sampling density. Of course the precise limit imposed by the noise to the effectively attainable resolution worsens exponentially with decreasing values of $r_{0}$, the reconstruction radius.

Figures 4-6 illustrate the importance of regularization in any practical situation with added noise. Here we vary the regularization parameter $\lambda$ to illustrate the tradeoff between the noise-filtering effects and the smoothing effects inherent to a Tikhonov regularization of the inversion. In Fig. 4, where regularization of $\lambda=0$ is used, the $5 \%$ Gaussiandistributed sampling noise entirely obscures the inversion. In

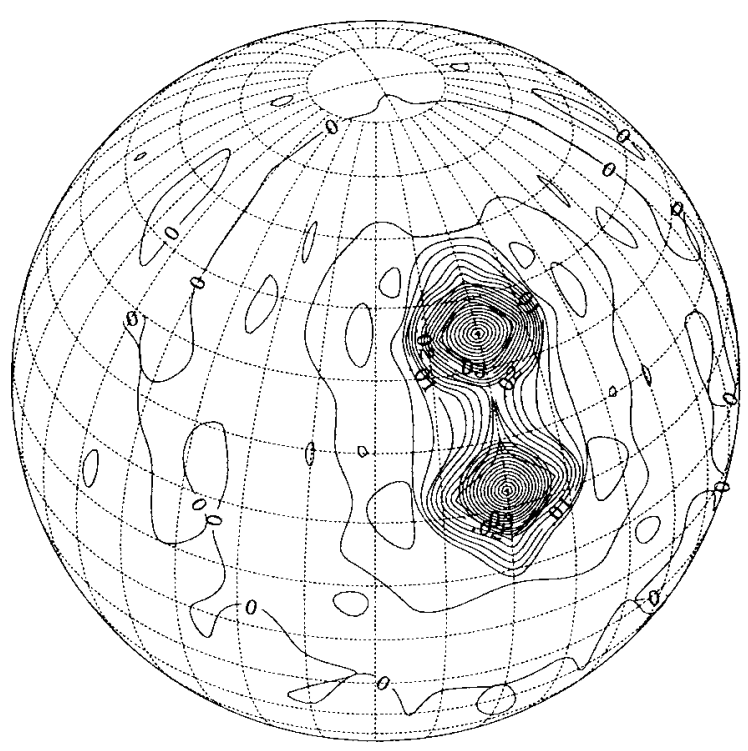

FIG. 2. Reconstruction with $N_{\text {lat }}=25, N_{\text {lon }}=48, S 2 N=$ inf, $e_{r}=0.195$. 


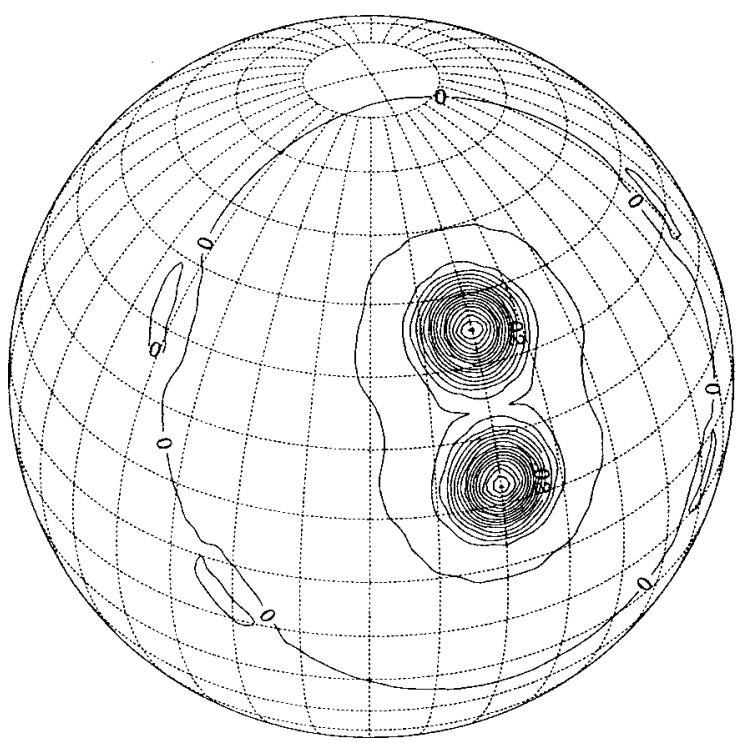

FIG. 3. Reconstruction with $N_{\text {lat }}=65, N_{\text {lon }}=128, S 2 N=\infty, e_{r}=3.34$ $\times 10^{-10}$.

Fig. 5 a value of $\lambda=0.03$ suppresses the noise but at a high cost to resolution. From Fig. 6 and Table II it appears that $\lambda=0.002$ is a good choice of a regularization parameter that balances noise amplification reduction against smoothing effects of the Tikhonov regularization. Table II shows that the same sampling as that used in the noiseless case appears to give the best error but this error value is considerably higher as compared to the noiseless case.

\section{CONCLUSIONS}

We have shown that for the idealized inverse magnetometry problem the determinable quantities in a VSH representation are moments of the expansion coefficients. Only one moment per coefficient is fixed by magnetic field measurements alone. This clearly exhibits the need for additional

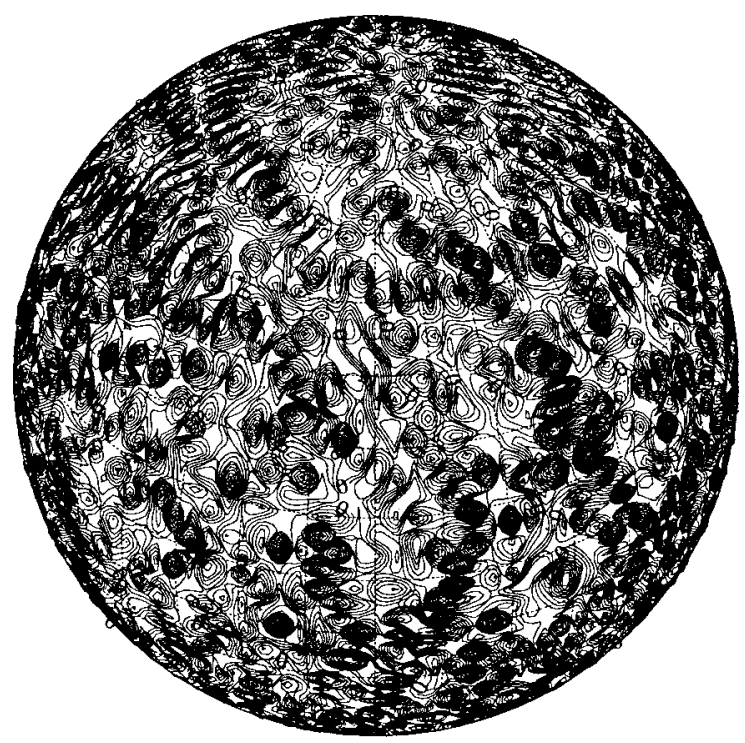

FIG. 4. Reconstruction with $N_{\text {lat }}=65, N_{\text {lon }}=128, S 2 N=20, \lambda=0, e_{r}$ $=71.9$.

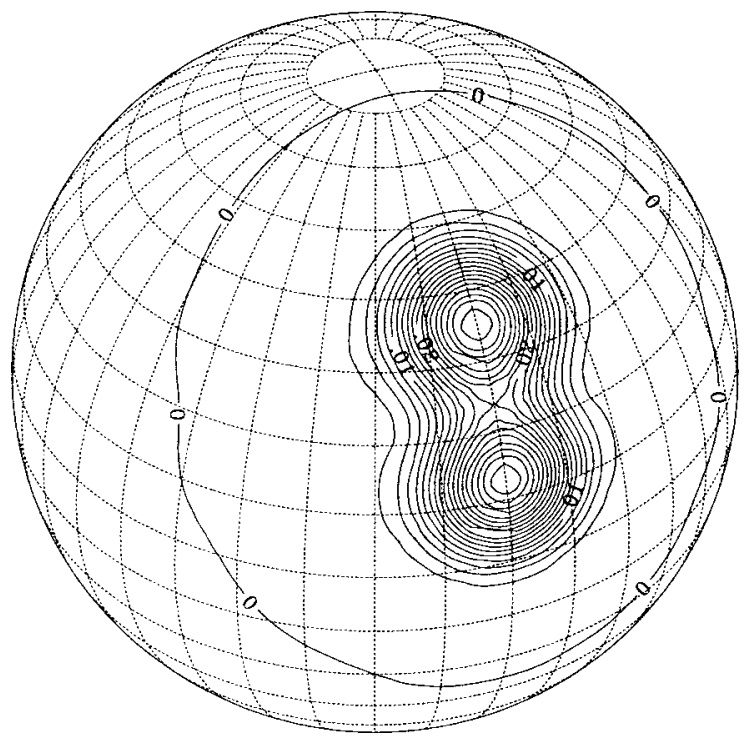

FIG. 5. Reconstruction with $N_{\text {lat }}=65, N_{\text {lon }}=128, S 2 N=20, \lambda=0.03, e_{r}$ $=0.400$.

trusted and testable constraints in the inverse magnetometry problem. In general these added constraints will clearly contain the majority of the information needed in an unique inversion for the current density of the idealized inverse magnetometry problem.

We have shown in what sense electric potential data may be incorporated to give additional information on the total current. When inverting for the total current, magnetic field data gives information about zeroth order near-field contributions to the current density, whereas electric potential data gives information about the first order contributions. When the conductivity is known, as in the case of a homogeneous conductor, both the magnetic and electric data give zeroth order information about the primary currents.

Finally we have shown that for a spherical shell a unique inversion is possible. However, the inversion has been shown

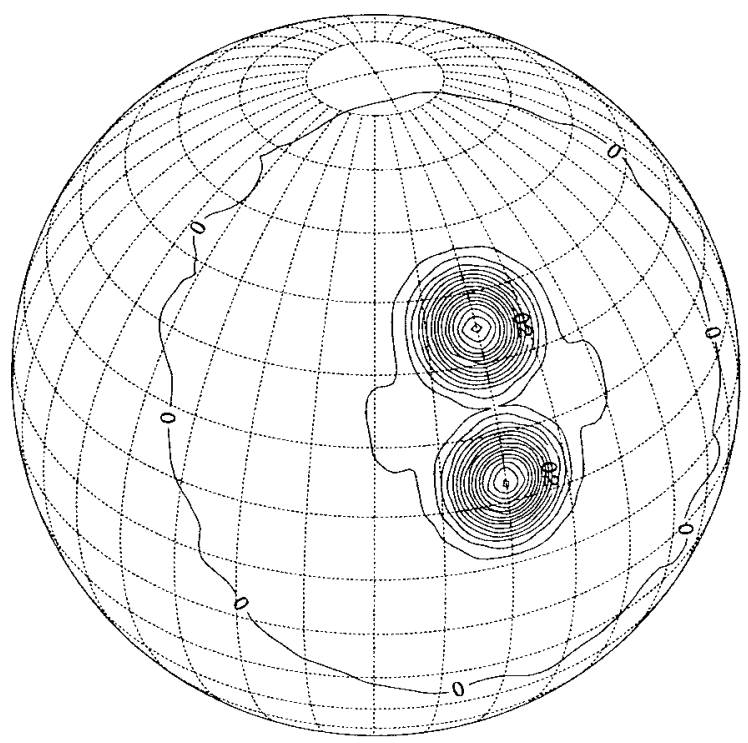

FIG. 6. Reconstruction with $N_{\text {lat }}=65, N_{\text {lon }}=128, S 2 N=20, \lambda=0.002, e_{r}$ $=9.60 \times 10^{-2}$. 
TABLE I. Relative error with $\mathrm{SNR}=\infty$.

\begin{tabular}{rrc}
\hline \hline$n_{\text {lat }}$ & $n_{\text {lon }}$ & \multicolumn{1}{c}{ Error } \\
\hline 9 & 16 & 0.633616464 \\
17 & 32 & 0.499727286 \\
25 & 48 & 0.194608761 \\
33 & 64 & 0.044827409 \\
41 & 80 & $5.70662263 \times 10^{-11}$ \\
49 & 96 & $6.27447749 \times 10^{-11}$ \\
57 & 112 & $1.05221049 \times 10^{-10}$ \\
65 & 128 & $3.34401624 \times 10^{-10}$ \\
\hline \hline
\end{tabular}

to be ill-conditioned in general. A regularization may be needed and this regularization must balance the filtering of amplified "high frequency" noise against the smoothing of spatial detail in the inversion.

\section{ACKNOWLEDGMENT}

The authors wish to thank the Mental Illness and Neuroscience Discovery Institute for their support of this work.

\section{APPENDIX A: DIVERGENCE CONSTRAINT}

In this Appendix we derive the restriction on the moments $m_{j m}^{j-1, j-1}=0$ given by the condition $\boldsymbol{\nabla} \cdot \mathbf{J}_{0}=0$. From this condition we have $\mathrm{e}^{10}$

$$
\begin{aligned}
& 0=\sqrt{j}\left(\frac{d}{d r}-\frac{j-1}{r}\right) a_{j m}^{j-1}(r), \\
& -\sqrt{j+1}\left(\frac{d}{d r}+\frac{j+2}{r}\right) a_{j m}^{j+1}(r) .
\end{aligned}
$$

Multiplying Eq. (A1) by $r^{k+2}$ and integrating by parts gives

$$
\begin{aligned}
0= & \sqrt{k+1}(k-j) \int_{0}^{1} r^{k-1} a_{j m}^{j+1}(r) r^{2} d r \\
& -\sqrt{j}(k-j+3) \int_{0}^{1} r^{k-1} \mathrm{a}_{j m}^{j-1}(r) r^{2} d r .
\end{aligned}
$$

since there is no current on the shell of radius 1 . Therefore, we obtain

$$
(k-j) m_{j m}^{j+1, k-1}+\sqrt{\frac{j}{j+1}}(k+j+1) m_{j m}^{j-1, k-1}=0,
$$

TABLE II. Relative error with $\mathrm{SNR}=20$.

\begin{tabular}{rrl}
\hline \hline$n_{\text {lat }}$ & $n_{\text {lon }}$ & \multicolumn{1}{c}{ Error } \\
\hline 9 & 16 & 0.633567187 \\
17 & 32 & 0.49996694 \\
25 & 48 & 0.199766954 \\
33 & 64 & 0.106897188 \\
41 & 80 & 0.096111095 \\
49 & 96 & 0.0965699241 \\
57 & 112 & 0.0960985884 \\
65 & 128 & 0.0960139612 \\
\hline \hline
\end{tabular}

which for $k=j$ becomes $m_{j m}^{j-1, j-1}=0$. It is easy to see that the condition $\boldsymbol{\nabla} \cdot \mathbf{A}_{0}=0$ is equivalent to $m_{j m}^{j-1, j-1}=0$, so the vanishing of the divergence of $\mathbf{A}_{0}$ is implied by that of $\mathbf{J}_{0}$ as expected.

\section{APPENDIX B: SPHERICAL HARMONIC PROPERTIES}

In this appendix we give some information on vector spherical harmonics. The vector spherical harmonics may be generated from the scalar spherical harmonics according to

$$
\begin{aligned}
\mathbf{Y}_{j m}^{j+1}= & \sqrt{\frac{j+1}{2 j+1}}\left(-\mathbf{e}_{r} Y_{j m}+\mathbf{e}_{\theta} \frac{1}{j+1} \frac{\partial Y_{j m}}{\partial \theta}\right. \\
& \left.+\mathbf{e}_{\phi} \frac{i m}{j+1} \frac{Y_{j m}}{\sin \theta}\right), \\
\mathbf{Y}_{j m}^{j}= & -\mathbf{e}_{\theta} \frac{m}{\sqrt{j(j+1)}} \frac{Y_{j m}}{\sin \theta}-\mathbf{e}_{\phi} \frac{i}{\sqrt{j(j+1)}} \frac{\partial Y_{j m}}{\partial \theta}, \\
\mathbf{Y}_{j m}^{j-1}= & \sqrt{\frac{j}{2 j+1}}\left(\mathbf{e}_{r} Y_{j m}+\mathbf{e}_{\theta} \frac{1}{j} \frac{\partial Y_{j m}}{\partial \theta}+\mathbf{e}_{\phi} \frac{i m}{j} \frac{Y_{j m}}{\sin \theta}\right) .
\end{aligned}
$$

In addition, the following property is used in this article:

$$
\mathbf{Y}_{j m}^{j}=\frac{-i}{\sqrt{j(j+1)}}\left(\mathbf{e}_{r} \times \nabla_{\Omega}\right) Y_{j m} .
$$

The vector spherical harmonics obey the orthogonality property

$$
\int_{0}^{\pi} \int_{0}^{2 \pi} \mathbf{Y}_{j^{\prime} m^{\prime}}^{* l^{\prime}}, \mathbf{Y}_{j m}^{l} \sin \theta d \theta d \phi=\delta_{j j^{\prime}} \delta_{l l^{\prime}} \delta_{m m^{\prime}}
$$

and the following relations for the divergence operator:

$$
\begin{aligned}
& \nabla \cdot\left[f \mathbf{Y}_{j m}^{j+1}\right]=-\sqrt{\frac{j+1}{2 j+1}}\left(\frac{d}{d r}+\frac{j+2}{r}\right) f Y_{j m}, \\
& \nabla \cdot\left[f \mathbf{Y}_{j m}^{j}\right]=0, \\
& \nabla \cdot\left[f \mathbf{Y}_{j m}^{j-1}\right]=\sqrt{\frac{j}{2 j+1}}\left(\frac{d}{d r}-\frac{j-1}{r}\right) f Y_{j m},
\end{aligned}
$$

and the curl operator ${ }^{10}$

$$
\begin{aligned}
\nabla \times\left[f \mathbf{Y}_{j m}^{j+1}\right]= & i \sqrt{\frac{j}{2 j+1}}\left(\frac{d}{d r}+\frac{j+2}{r}\right) f \mathbf{Y}_{j m}^{j}, \\
\nabla \times\left[f \mathbf{Y}_{j m}^{j}\right]= & i \sqrt{\frac{j}{2 j+1}\left(\frac{d}{d r}-\frac{j}{r}\right) f \mathbf{Y}_{j m}^{j+1}} \\
& +i \sqrt{\frac{j+1}{2 j+1}}\left(\frac{d}{d r}+\frac{j+1}{r}\right) f \mathbf{Y}_{j m}^{j-1}, \\
\nabla \times\left[f \mathbf{Y}_{j m}^{j-1}\right]= & i \sqrt{\frac{j+1}{2 j+1}}\left(\frac{d}{d r}-\frac{j-1}{r}\right) f \mathbf{Y}_{j m}^{j},
\end{aligned}
$$

where $f=f(r)$. We also give the connection between the spherical harmonics and associated Legendre functions

$$
Y_{j m}(\theta, \phi)=\sqrt{\frac{2 j+1}{4 \pi} \frac{(j-m) !}{(j+m) !}} P_{j}^{m}(\cos \theta) \exp i m \phi .
$$




\section{APPENDIX C: ROTATION OF ELEMENTAL MAGNETIC FIELDS}

In Sec. VII we performed rotations upon elemental fields to obtain our simulated fields. Each elemental field $B_{r}$ is a sum of Legendre polynomials of the form $B_{r}$ $=\Sigma_{l} \alpha_{l} P_{l}(\cos \theta)$. The rotation operator $\mathcal{R}_{r}=\mathcal{R}\left(\phi_{r}, \theta_{r}\right)$, acting on a Legendre polynomial $P_{l}(\cos \theta)$, yields

$$
\begin{aligned}
\mathcal{R}_{r} P_{l}(\cos \theta)= & P_{l}\left(\theta_{r}\right) P_{l}(\theta)+2 \sum_{m=1}^{l} \frac{(l-m) !}{(l+m) !} P_{l}^{m}\left(\theta_{r}\right) \\
& \times P_{l}^{m}(\theta) \cos m\left(\phi_{r}-\phi\right)
\end{aligned}
$$

so that the rotated elemental field becomes

$$
\begin{aligned}
B_{r}(\theta, \phi)= & \sum_{l} \alpha_{l} P_{l}\left(\theta_{r}\right) P_{l}(\theta)+2 \sum_{m l} \alpha_{l} \frac{(l-m) !}{(l+m) !} P_{l}^{m}\left(\theta_{r}\right) \\
& \times P_{l}^{m}(\theta) \cos m\left(\phi_{r}-\phi\right) .
\end{aligned}
$$

The fields due to each elemental current can then be added at the sampling points for the simulated field measurement.

\section{APPENDIX D: TIKHONOV REGULARIZATION}

In this Appendix we give a brief review of Tikhonov regularization as it applies to this article. ${ }^{15}$ We assume one wants to invert the following linear equation given the data $d$

$$
\text { Af }=d \text {. }
$$

We will also assume that the matrix $A$ is ill conditioned. Tikhonov regularization of Eq. (D1) obtains a wellconditioned solution by minimizing the following functional:

$$
\hat{f}_{\lambda}=\arg \min \left\{\lambda^{2}\left\|L\left(f-f^{\infty}\right)\right\|^{2}+\|d-A f\|^{2}\right\},
$$

where $\lambda$ is the regularization parameter and $f^{\infty}$ is a default solution. If $\lambda$ is large the data misfit term, $\|d-A f\|^{2}$, be- comes negligible and the solution tends to $f^{\infty}$ in which high frequency behavior has been effectively smoothed, regardless of its origin. If $\lambda$ is small the solution tends to the solution of Eq. (D1), which is presumably ill conditioned, resulting in noise amplification. A practical choice of the regularization parameter balances smoothing of the solution against reduction of the amplified high frequency noise in the data. Minimizing Eq. (D2) one obtains

$$
\left(\lambda^{2} L^{t} L+A^{t} A\right) f=\lambda^{2} L^{t} L f^{\infty}+A^{t} d .
$$

For systems of equations which are relatively small (a few hundred equations) this equation may be directly solved for $f$. For larger systems one may employ searching or iterative algorithms.

\footnotetext{
${ }^{1}$ H. von Helmholtz, Ann. Phys. Chem. 89, 211 (1853).

${ }^{2}$ J. Sarvas, Phys. Med. Biol. 32, 11 (1987).

${ }^{3}$ J. Malmivuo and R. Plonsey, Bioelectromagnetism (Oxford University Press, Oxford, 1995).

${ }^{4}$ B. Roth, N. G. Sepulveda, and J. P. Wikswo, J. Appl. Phys. 65, 361 (1989)

${ }^{5}$ S. K. Law, Brain Topagr. 6, 99 (1993).

${ }^{6}$ L. A. Geddes and L. E. Baker, Med. Biol. Eng. 5, 271 (1967)

${ }^{7}$ S. B. Baumann, D. R. Wonzy, S. K. Kelly, and F. M. Meno, IEEE Trans. Biomed. Eng. 44, 220 (1997).

${ }^{8}$ M. Hämäläinen, R. Hari, R. J. Ilmoniemi, J. Knuutila, and O. V. Lounasmaa, Rev. Mod. Phys. 65, 413 (1993).

${ }^{9}$ A. S. Fokas, I. M. Gel'fand, and Y. Kurylev, Inverse Probl. 12, L9 (1996).

${ }^{10}$ D. A. Varshalovich, A. N. Moskalev, and V. K. Khersonskii, Quantum Theory of Angular Momentum (World Scientific, Singapore, 1989).

${ }^{11}$ J. C. Adams and P. Swarztrauber, Mon. Weather Rev. 127, 1872 (1999).

${ }^{12}$ P. Swarztrauber, SIAM (Soc. Ind. Appl. Math.) J. Numer. Anal. 16, 934 (1979).

${ }^{13}$ W. H. Press, S. A. Teukolsky, W. T. Vetterling, and B. P. Flannery, Numerical Recipes in $C$, 2nd ed. (Cambridge University Press, New York, 1992).

${ }^{14}$ J. P. Boyd, Chebyshev and Fourier Spectral Methods, 2nd ed. (Dover, Mineola, N.Y., 2001).

${ }^{15}$ H. W. Engl, M. Hanke, and A. Neubauer, Regularization of Inverse Problems (Kluwer, Boston, 2000).
} 